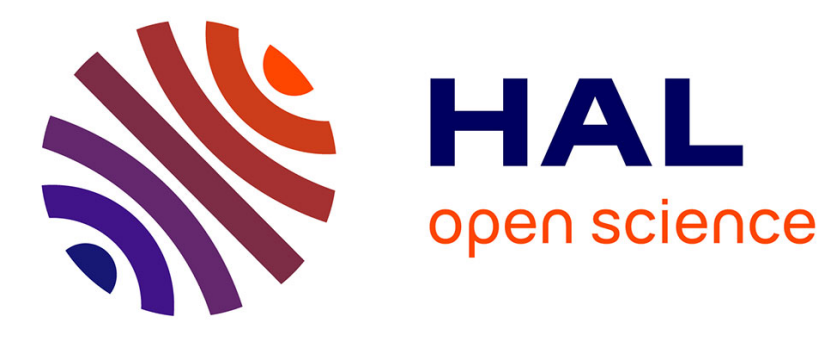

\title{
Nc-AFM Imaging and Manipulation of a Triphenylene Derivative on $\operatorname{KBr}(001)$
}

Véronique Langlais, Olivier Guillermet, David Martrou, André Gourdon, Sebastien Gauthier

\section{- To cite this version:}

Véronique Langlais, Olivier Guillermet, David Martrou, André Gourdon, Sebastien Gauthier. NcAFM Imaging and Manipulation of a Triphenylene Derivative on $\operatorname{KBr}(001)$. Journal of Physical Chemistry C, 2016, 120 (32), pp.18151-18157. 10.1021/acs.jpcc.6b06113 . hal-01715332

\section{HAL Id: hal-01715332 \\ https://hal.science/hal-01715332}

Submitted on 18 May 2018

HAL is a multi-disciplinary open access archive for the deposit and dissemination of scientific research documents, whether they are published or not. The documents may come from teaching and research institutions in France or abroad, or from public or private research centers.
L'archive ouverte pluridisciplinaire HAL, est destinée au dépôt et à la diffusion de documents scientifiques de niveau recherche, publiés ou non, émanant des établissements d'enseignement et de recherche français ou étrangers, des laboratoires publics ou privés. 


\section{nc-AFM imaging and manipulation}

\section{of a triphenylene derivative on $\mathrm{KBr}(001)$}

V. Langlais ${ }^{a}$, O. Guillermet ${ }^{a, b}$, D. Martrou ${ }^{a}$, A. Gourdon $^{a}$, S. Gauthier $* a$

(a) NanoSciences Group, CEMES, CNRS UPR 8011, 29 rue J. Marvig, 31055 Toulouse (France)

(b) Université de Toulouse, UPS, 29 rue J. Marvig, 31055 Toulouse (France)

* Corresponding author: gauthier@ cemes.fr 


\begin{abstract}
The adsorption of single hexamethoxyltriphenylene molecules on $\mathrm{KBr}(001)$ at low temperature $(\mathrm{T}<10 \mathrm{~K})$ was studied by low temperature $(\mathrm{T}=5 \mathrm{~K})$ non-contact atomic force microscopy in ultra-high vacuum. Images of the frequency shift, measured at constant height, show intramolecular features that are interpreted on the basis of molecular force-field calculations. The main conclusion is that the experimental contrast originates from the electrostatic interaction between a negatively terminated ionic tip and the atomic charges of the molecule. The main trends of the experimental observations of lateral manipulations could also be reproduced by the same method, giving some insight into the manipulation mechanisms. In particular, it is shown that the strong interaction of the oxygen atoms of the molecule with positive ions of the tip plays a major role by facilitating the partial lifting of the molecule from the substrate during the intermediate stages of these manipulation processes.
\end{abstract}




\section{INTRODUCTION}

Using single adsorbed molecules to perform elementary electronic functions or calculations is the main purpose of molecular electronics. But a precise control of a single molecule-based device requires to image the molecule. Imaging single molecules is now performed routinely by STM, which necessitates conducting surfaces (metals, semiconductors or ultrathin insulating layers on conducting substrates). This condition constitutes a severe limitation and in most cases using molecules adsorbed on bulk insulators would greatly simplify the device design and operation.

Due to the continuous development of Atomic Force Microscopy in non-contact mode (ncAFM), the number of studies devoted to the adsorption of molecular layers or islands on bulk insulators is steadily increasing [1-13]. Nevertheless, only very few investigations of single molecules adsorbed on bulk insulators are reported until now, because, at room temperature, most molecules tend to diffuse on these substrates. No isolated molecules could be observed at room temperature so far except when trapped on step edges $[3,4]$.

At low temperature, where diffusion is inhibited, the adsorption of Co-Salen on $\mathrm{NaCl}(001)$ [2] and $\mathrm{NiO}(001)$ [9] at $8 \mathrm{~K}$ has been studied in detail by nc-AFM from constant $\Delta \mathrm{f}$ images. Based on these experiments, the interest of using metallic tips on this type of systems was pointed out.

Here, we report on a study on individual isolated hexamethoxytriphenylene molecules (HMTP: Fig 1a) on the (001) surface of the bulk insulator KBr. This study is performed in UHV, at low temperature (5K). This system was already studied at room temperature [5].

In the following, after describing our experimental methods, we present nc-AFM frequency shift images of isolated HMTP obtained in the constant height mode. These images display submolecular contrast that we interpret on the basis of force-field-based numerical simulations. The same methods are then applied to understand why and in which conditions 
isolated HMTP molecules are manipulated (laterally or vertically) by the tip. This analysis is followed by a discussion and conclusions.

\section{METHODS}

\section{Experimental}

The $\mathrm{KBr}$ crystal was cleaved in air just before being introduced in the UHV system. It was annealed at $150^{\circ} \mathrm{C}$ for 1 hour in order to remove residual charges originating from the cleavage process. The sample was then transferred to the low temperature (5K) STM/nc-AFM (Scienta Omicron). HMTP molecules, in powder form, were then deposited on the cooled $(\mathrm{T}<10 \mathrm{~K})$ surface from a heated crucible. The force sensor was a tuning fork of the qPlus sensor type with resonance frequency $\mathrm{f}_{0}$ and $\mathrm{Q}$ factor of the order of $25,000 \mathrm{~Hz}$ and 20,000 respectively. It was equipped with a glued electrochemically etched tungsten tip. The control electronics were provided by SPECS. All images, spectra, and manipulation experiments were performed at a temperature of $5 \mathrm{~K}$. All the displayed experimental images are oriented such that the vertical and horizontal directions correspond to $<001>$ non polar crystallographic directions of the $\mathrm{KBr}$ surface.

\section{Numerical}

The COMPASS (Condensed-phase Optimized Molecular Potentials for Atomistic Simulation Studies) force-field [14] was used with Materials Studio [15] to optimize the conformation of an isolated molecule on $\operatorname{KBr}(001)$ in the presence of the tip. This force-field is well suited to calculate the adsorption of organic molecules on the surface of inorganic materials. It has been parameterized using condensed phase properties as well as ab initio and empirical data for isolated molecules [16,17]. Previous studies have shown that the interaction of organic molecules with ionic surfaces is dominated by van der Waals and electrostatic forces. In 
addition, these works indicate that the charge transfer between the substrate and the molecule is generally negligible for this type of surfaces [18], ensuring that force-field based calculations are sufficient for a realistic description of these systems.

\section{RESULTS}

NC-AFM imaging of HMTP molecules on the $\operatorname{KBr}(001)$ surface

\section{a. Constant $\Delta \mathrm{f}$ images}

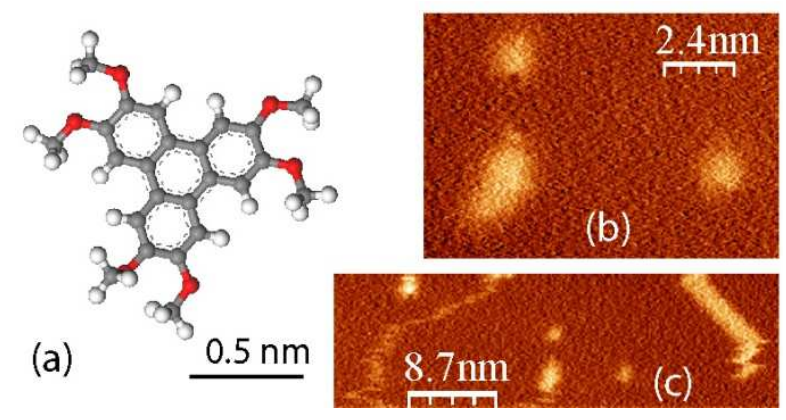

Figure 1: (a) HMTP optimized in vacuum. Oxygen atoms are depicted in red, carbon in grey and hydrogen in white. (b) and (c) Constant $\Delta f$ images of HMTP/KBr(001). (b) Amplitude $A=150 \mathrm{pm}, \Delta f=-4.1 \mathrm{~Hz},(\mathrm{c}) A=250 \mathrm{pm}, \Delta f=-2.1 \mathrm{~Hz}$.

A constant $\Delta \mathrm{f}$ image of HMTP molecules at low coverage on $\mathrm{KBr}(001)$ is displayed in Fig. 1b. Single molecules appear as circular, featureless objects, with a mean diameter of $\approx 1.3 \mathrm{~nm}$ and a height of $\approx 0.18 \mathrm{~nm}$. These dimensions are compatible with the molecular model shown in Fig. 1a. The bigger object could originate from an aggregate of a few molecules. The image of the single molecules do not show any intra-molecular resolution. Trying from the imaging conditions of Fig. $1 \mathrm{~b}$ to improve the resolution by optimizing either $\Delta \mathrm{f}$ or the amplitude A led to molecular manipulation, where the molecules are laterally displaced by the scanning tip as illustrated in Fig. 1c. 


\section{b. Constant height images}

The constant height mode, which produces $\Delta \mathrm{f}$ images, is increasingly used since the pioneering work of Gross et al [19] on the high resolution imaging of pentacene $/ \mathrm{NaCl} / \mathrm{Cu}(111)$ at low temperature. It is also frequently used at room temperature (see for instance [13]). Its advantages are that one of the feedback loops of nc-AFM is disabled, simplifying the settings of the instrument and, more importantly, the tip can approach or go beyond the minimum of the $\Delta f(Z)$ curve without risking a tip crash, in contrast to the more conventional constant $\Delta \mathrm{f}$ mode. While the constant height mode is generally restricted to very flat samples, it has also been used efficiently to image the top facet of clusters [20].

Fig. 2 displays constant height $\Delta \mathrm{f}$ images of 3 single HMTP molecules at different heights $\mathrm{Z}$. The evolution of the image of HMTP-A when $\mathrm{Z}$ decreases is characterized by the apparition of a nearly circular black depression, with a diameter that increases from $1 \mathrm{~nm}$ to $1.4 \mathrm{~nm}$, on which submolecular clearer features emerge, reaching positive frequency shifts for the smaller Z. HMTP-B and C follow the same trends. This behavior is similar to the observations reported by Gross et al [19], where pentacene molecules where imaged at constant height with a CO-terminated tip. The black depression was associated with long range attractive electrostatic and van der Waals interactions, while the submolecular features were interpreted in terms of Pauli repulsive interactions. Despite these similarities, the situation is different here: the tip is not intentionally functionalized, and the size of the submolecular features does not go down to atomic resolution as in [19].

Beyond this general behavior, these images also share common features such as minima ( 2 for HMTP-A, 2 to 4 for HMTP-B, 2 to 3 for HMTP-C) with quite similar topologies. But they are not identical. Note that each of these series of images was obtained with the same qPlus 
sensor, but with different tip apices since the tip was often reconditioned by gentle contacts with the surface while measuring $\Delta \mathrm{f}(\mathrm{Z})$ approach curves.

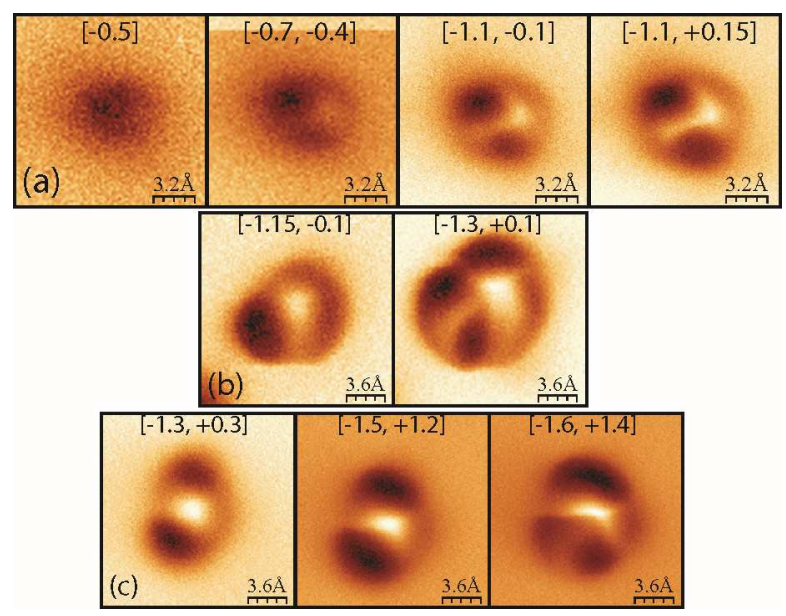

Figure 2: Constant height $4 f$ images of 3 HMTP molecules at different height Z. Experimental conditions: $\operatorname{HMTP}(a) Z=Z_{0}\left(\Delta f_{0}=-3.1 \mathrm{~Hz}\right), Z=Z_{0^{-}} 40 \mathrm{pm}, Z=Z_{0^{-}} 60 \mathrm{pm}, Z=Z_{0^{-}}$ $80 \mathrm{pm} . H M T P(b) Z=Z_{0}\left(\Delta f_{0}=-2.7 \mathrm{~Hz}\right), Z=Z_{0}-100 \mathrm{pm} . H M T P(c) Z=Z_{0}\left(\Delta f_{0}=-3.7 \mathrm{~Hz}\right), Z=Z_{0}-30$ pm, $Z=Z_{0}-60 \mathrm{pm} . Z_{0}$ is the height of the tip in the constant $\Delta f$ mode on $\mathrm{KBr}$ with the setpoint $\Delta f_{0}$ indicated between parentheses. Amplitude $A=150 \mathrm{pm}$. The $\Delta f$ scale has been translated in such a way that its origin corresponds to the $\mathrm{KBr}$ substrate, in order to approximately remove the long range van der Waals contribution. The values between brackets indicate the $\Delta f$ minimum and maximum values on the molecule.

In the following, we try to interpret the contrasts observed on the images of Fig.2. More specifically, we address the following questions:

Which interactions are involved in the formation on the contrast?

Why is the contrast different from one molecule to another?

Can we extract information about the tip? 
We have two objectives in mind. The first one is to get as much information as possible on the adsorption of HMTP on $\operatorname{KBr}(001)$. The second, more methodological, consists in identifying the experimental factors that limit the spatial resolution of nc-AFM on this type of system.

\section{Modeling of HMTP on $\mathrm{KBr}(001)$}

The purpose of this modeling is not to reproduce precisely the experimental results. One of the main obstacles toward this objective is to determine the precise structure of the tip, a huge task which has been achieved in only a few cases (see for instance [21]). It requires to perform calculations with a large number of plausible tip structures until an acceptable fit with the experimental results is achieved.

Here, following the pioneering work reported in [22], we will consider that the tip is constituted of a $\mathrm{KBr}$ cluster linked to the $\mathrm{W}$ tip of the qPlus sensor. Indeed, it is generally accepted that when imaging the surface of ionic crystals, in particular $\mathrm{KBr}$, the tip is rapidly covered by material that has been picked up on the sample surface [23]. The structure of the tip is kept as simple as possible, being modelled by a $\mathrm{KBr}$ cube of 8 atoms oriented with its diagonal perpendicular to the sample surface with either a $\mathrm{Br}$ ion or a $\mathrm{K}$ ion pointing toward the surface. It was checked that including more ions in the tip, for instance 36 atoms as shown in Supporting Information (SI) Figure S2, does not significantly change the conclusions of our study.

The $\mathrm{KBr}$ slab was four (001) atomic layers thick. The surface was constituted by $6 \times 6$ conventional unit cells, with a vacuum slab of $4 \mathrm{~nm}$ to avoid any coupling between neighboring supercells due to the periodic boundary conditions. The van der Waals and electrostatic energies were calculated using the Ewald summation method. 


\section{Adsorption geometry of HMTP on $\operatorname{KBr}(001)$}

The minimum energy conformation obtained by calculation is illustrated in Fig. 3. For this calculation the two upper layers of the slab as well as the molecule were allowed to relax. The adsorption energy is $\mathrm{E}_{0}=0.87 \mathrm{eV}$. The molecule binds to the surface by positioning two of its oxygen atoms directly above the two $\mathrm{K}^{+}$ions colored in green in Figure 3, with a $\mathrm{K}^{+}-\mathrm{O}$ distance $\approx 0.3 \mathrm{~nm}$, while the other four oxygen atoms are distant by more than $\approx 0.38 \mathrm{~nm}$ from any $\mathrm{K}+$ ion.

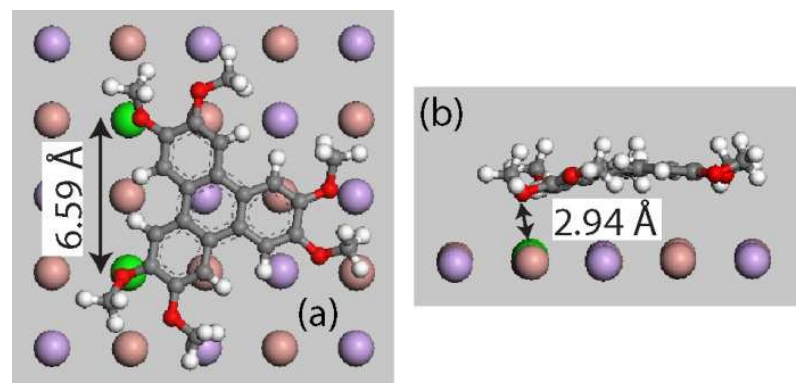

Figure 3: (a) Top and (b) side view of HMTP in its minimum energy configuration on $\mathrm{KBr}(001) . \mathrm{K}^{+}$ions are depicted in violet, $\mathrm{Br}$ ions in pale red. The two $\mathrm{K}^{+}$ions on which the molecule is more strongly electrostatically linked are in green

As can be seen on the side view in Figure $3 b$, this electrostatic interaction bends the central aromatic part of the molecule downwards. This constitutes another example of a adsorption stabilized by "charge matching" as already illustrated for instance for another triphenylene derivative [7] or for functionalized truxenes [3] on $\operatorname{KBr}(001)$.

Figure 4 displays the van der Waals surface of HMTP colored according to its molecular electrostatic potential. As expected, the oxygen atoms are surrounded by a negative potential while the methyl groups are surrounded by a positive potential. The charge matching derives from the nearly perfect equality between the distance separating the two binding oxygen atoms of the molecule $(0.69 \mathrm{~nm}$, Figure 4$)$ and the distance between the two second nearest 
$\mathrm{K}^{+}$ions of the substrate $(0.66 \mathrm{~nm}$, Figure $3 \mathrm{a})$ onto which the molecule is electrostatically bound.

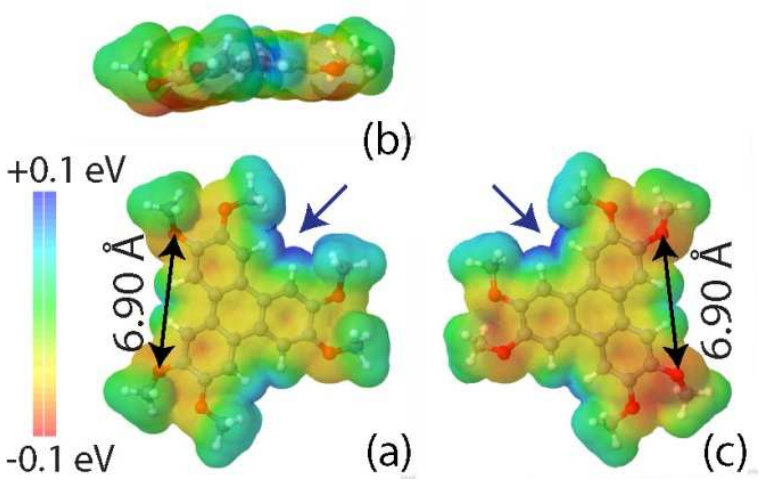

Figure 4: Molecular van der Waals surface of HMTP colored according to its electrostatic potential. The viewpoint for $(a)$ and $(b)$ is the same as for Figure $3 a$ and $b$ respectively while (c) is seen from the $\mathrm{KBr}$ surface.

\section{Calculation of $\Delta \mathbf{f}$ maps}

In a first step, the total energy $\mathrm{E}$ of the system constituted by the molecule in its optimized conformation on the substrate in interaction with the tip was calculated, without any further relaxation. This calculation was performed for each position $(x, y, z)$ of the tip. The tipsurface force $F(x, y, z)=-\frac{\partial E}{\partial z}$ and its derivative relative to $\mathrm{z}$ were evaluated by numerical differentiation. $\Delta f(x, y, z)$ was then calculated by numerical integration of the relation derived by Giessibl [24]:

$$
\Delta f(x, y, z)=-\frac{2 \pi f_{0}^{2}}{k A} \int_{0}^{1 / f_{0}} d t F\left[\left(x, y, D+A \sin \left(2 \pi f_{0} t\right)\right] A \sin \left(2 \pi f_{0} t\right)\right.
$$

where $\mathrm{D}$ is the distance between the front tip ion and the substrate when $\mathrm{A}=0$.

$\Delta \mathrm{f}$ images calculated for a $\mathrm{Br}^{-}$tip or a $\mathrm{K}^{+}$tip are displayed in Fig. 5 and 6 respectively. Fig. 5c and $6 \mathrm{c}$ are obtained from the force derivative map according to the formula: 


$$
\lim _{A \rightarrow 0} \Delta f=-\frac{f_{0}}{2 k} \frac{\partial F}{\partial z}
$$

giving the small amplitude limit of equation (1). As expected, these images are nearly identical to the images at $\mathrm{A}=10 \mathrm{pm}$.

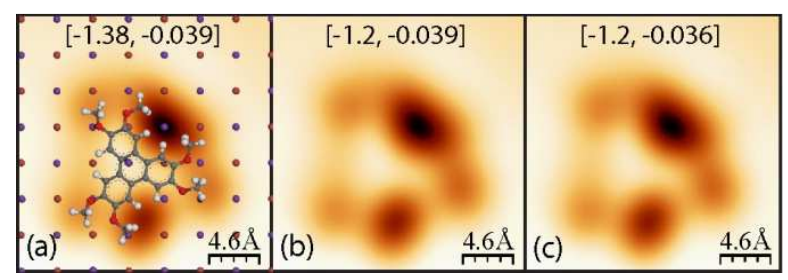

Figure 5: $\Delta f$ images calculated with a $\mathrm{Br}^{-}$tip at $D=11 \AA$. . (a) $A=150 \mathrm{pm}$, (b) $A=10 \mathrm{pm}$, (c) force derivative.

The images calculated with the experimental amplitude $A=150 \mathrm{pm}$ are very similar to the images calculated with $\mathrm{A}=10 \mathrm{pm}$. This is a first evidence that the image contrast formation arises from long range interaction, in contrast to the case of atomic resolution mentioned previously. In addition, the contrast obtained with the $\mathrm{K}^{+}$tip is qualitatively the opposite of the contrast obtained with the $\mathrm{Br}^{-}$tip. This observation clearly indicates that the image is dominated by the long range electrostatic interaction in the selected $\mathrm{D}$ range.

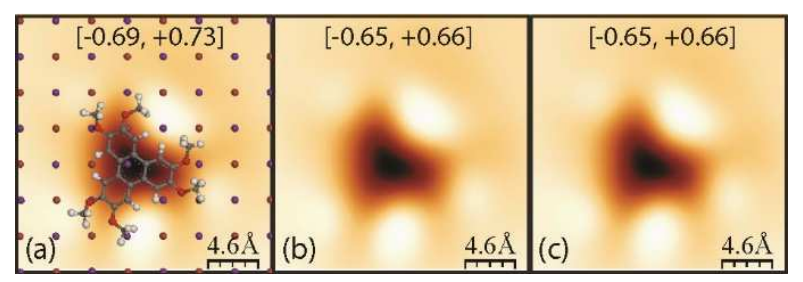

Figure 6: $\Delta f$ image calculated with a $K^{+}$tip at $D=11 \AA$. (a) $A=150 \mathrm{pm}$, (b) $A=10 \mathrm{pm}$, (c) force derivative. 
It is immediately obvious that the calculation with the $\mathrm{K}^{+}$tip does not compare favorably with the experimental images of Figure 2. In contrast, a fair qualitative agreement between the $\mathrm{Br}^{-}$ tip images and the experimental images is obtained, especially for HMTP-C, which displays, like in the calculation a unique maximum with two marked minima. Thus, we conclude that the tip used in the experiment was of the $\mathrm{Br}^{-}$type. This conclusion is also consistent with the electrostatic potential map of Fig.4a: the negatively polarized tip is attracted by the positive (blue) regions, resulting in the two minima, while it is repelled by the central more negative (yellow) region. In addition, the electrostatic potential map shows that the appearance of the minima is closely related with the orientation of the $\mathrm{O}-\mathrm{CH}_{3}$ bounds in the molecule. For instance, the potential is more positive when these bounds point toward the outside of each $\mathrm{O}$ pair, as indicated by the blue arrows in Figure $4 \mathrm{a}$ and $\mathrm{c}$.

Note that this comparison also excludes a metallic tip, since in most cases the polarity of these tips is positive, i.e. their associated dipole is pointing outside $[25,26]$.

The van der Waals and electrostatic contributions to the $\Delta \mathrm{f}$ image of the minimum energy configuration of HMTP on $\operatorname{KBr}(001)$ are displayed in Figure 7. The van der Waals image only exhibits attractive interaction. In contrast both attractive and repulsive interactions contribute to the electrostatic image. The intramolecular contrast is therefore purely of electrostatic origin.

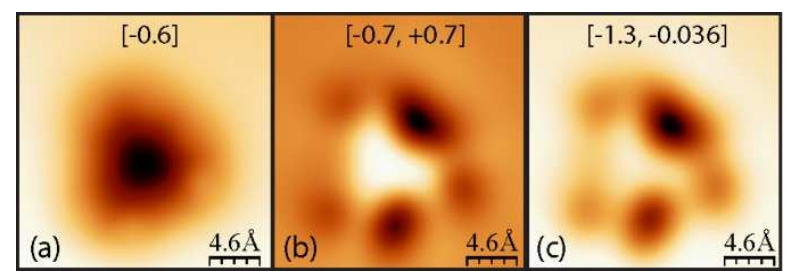

Figure 7: (a) van der Waals and (b) electrostatic contribution to (c) the $4 f$ image of the minimum energy configuration of HMTP on $\mathrm{KBr}(001)$. 
Figure 8 shows, in addition to the minimum energy configuration already discussed, two other conformations of slightly lower adsorption energies that were obtained by optimizing the system after quenching a molecular dynamics simulation. They correspond to local relative minima of the potential energy. These conformations can be distinguished by the orientation of their methyl groups: conformation (b) is obtained from (a) by rotating around their CO bond the 2 upper methyl groups (surrounded by a blue ellipse in Figure $8 \mathrm{a}$ and b) while conformation (c) is obtained from (a) by rotating the lower right methyl group (marked by a red circle in Figure 8a and c). This last change induces a slight rotation of the whole molecule, which ends in a fully symmetric situation, aligned along a non-polar [100] direction of $\operatorname{KBr}(001)$.

Even if the images still share the common features mentioned previously, the location of the methyl groups has a noticeable influence: by changing the relative altitude of the central aromatic part, the oxygen atoms and the methyl groups, they alter the relative intensity of the maxima and minima of the images.

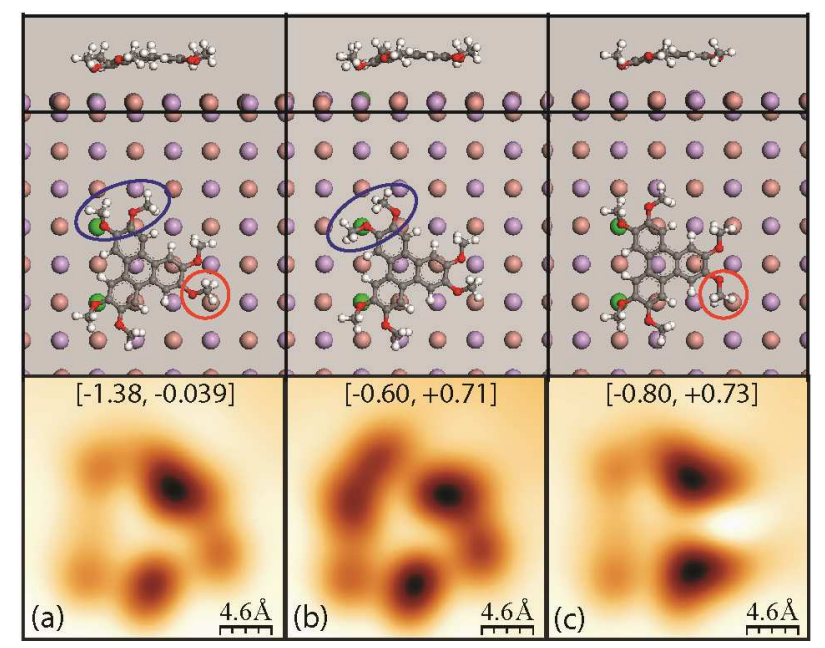

Figure 8: Side and top views and the corresponding images for the three lowest energy calculated configurations for $H M T P / K B r(001)$. The adsorption energies are $E_{a d s}=E_{0}=0.87 \mathrm{eV}$ for (a), Eads $=E_{0}-11 \mathrm{meV}$ for $(b)$ and Eads $=E_{0}-15 \mathrm{meV}$ for $(\mathrm{c})$. 
The influence of the size of the tip and its orientation (tilt of the tip axis or rotation along an axis perpendicular to the surface) was also checked. The results, reported in Supporting Information Figure S2 and S3, only show a limited influence of these parameters.

To go further, the same kind of calculations were performed allowing the molecule and the first atomic layer of the $\mathrm{KBr}$ substrate to relax at each position of the tip. Three behaviors can be distinguished, depending on D. When the tip is far away from the surface, the relaxations of the molecule and the substrate induced by the tip are very small and do not affect the images. In the intermediate range, the molecule is reversibly displaced from its equilibrium position in the absence of the tip. These displacements can be quite large $(\sim 50 \mathrm{pm})$ and make the method for calculating the images previously described inadequate. A realistic modelling would require molecular dynamics simulation at each point, which is beyond the scope of this paper. For shorter D distances, irreversible displacements occur: the molecule is laterally or even vetically manipulated by the tip.

\section{Manipulations}

Reducing the tip-substrate distance from the stable imaging conditions used in Figure 2 led quite often to lateral molecular manipulation, as illustrated in the constant height $\Delta \mathrm{f}$ image of Figure 9. The molecule is displaced along a polar [110] direction of the substrate, as quite often observed on alkali halide substrates. The $\Delta \mathrm{f}$ profile along the manipulation path, displayed in Figure $9 \mathrm{~b}$ shows that the molecule proceeds by jumps of $0.47 \mathrm{~nm}$ length, corresponding to the nearest neighbor distance along <110> directions. The shape of this profile suggests that the molecule is repelled by the tip: in the first part of the elementary manipulation step, the tip images the molecule until a sudden jump happens, corresponding to the translation of the molecule to the next equivalent site of the trajectory. This is typical of a 
"pushing" mode [27-29]. Note that it was necessary to lift the tip by $50 \mathrm{pm}$ to release the molecule, as shown on the $\mathrm{Z}$ profile in Figure $9 \mathrm{~b}$.

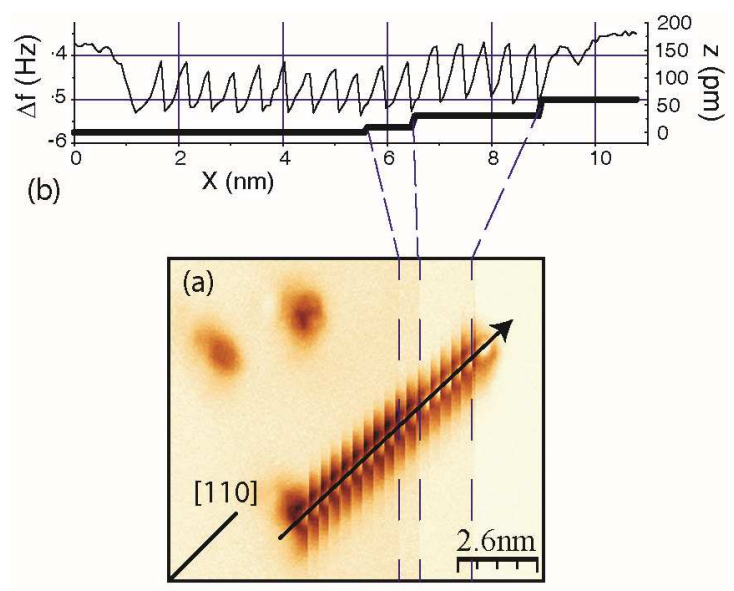

Figure 9: (a) Constant height $\triangle f$ image of a HMTP manipulation sequence. (b) Profile along the manipulation path [black arrow in (a)]. The fast scanning direction is vertical. $A=150$ pm.

This behavior could be reproduced by simulations. Here, only the surface layer and the molecule were allowed to relax. A complete calculated sequence is shown and discussed in SI (V1 and V2 video files). The images of Figure 10 are extracted from this sequence. The closest tip-surface distance is given by $\mathrm{D}-\mathrm{A}=0.66 \mathrm{~nm}$. Between Figure 10a and 10f, the molecule is translated between two nearest neighbor sites along the [110] direction.

The tip always stays on the left of the molecule, meaning that the manipulation proceeds indeed by pushing. A careful examination of the manipulation sequence (SI) shows that one of the oxygen atoms of the molecule (O1 in Figure 10a) interacts strongly with the closest $\mathrm{K}^{+}$ ions of the tip second layer (KTip in Figure 10a). This bond allows the tip to lift one of the branches of the molecule (Figure $10 \mathrm{c}$ and d), facilitating the displacement toward the next site along the [100] substrate direction. 
The length of this dO1-Ktip bond goes down to $0.27 \mathrm{~nm}$. This corresponds to the equilibrium distance between an isolated $\mathrm{K}+$ ion and an oxygen atom of the molecule, as given by the numerical simulation. This short distance indicates that the $\mathrm{O} 1$ is strongly bound to the tip that pushes on the molecule as suggested by the images of Figure 10. It means that, for manipulation, in contrast to imaging, the repulsive short-range part of the van der Waals interaction plays a significant role.

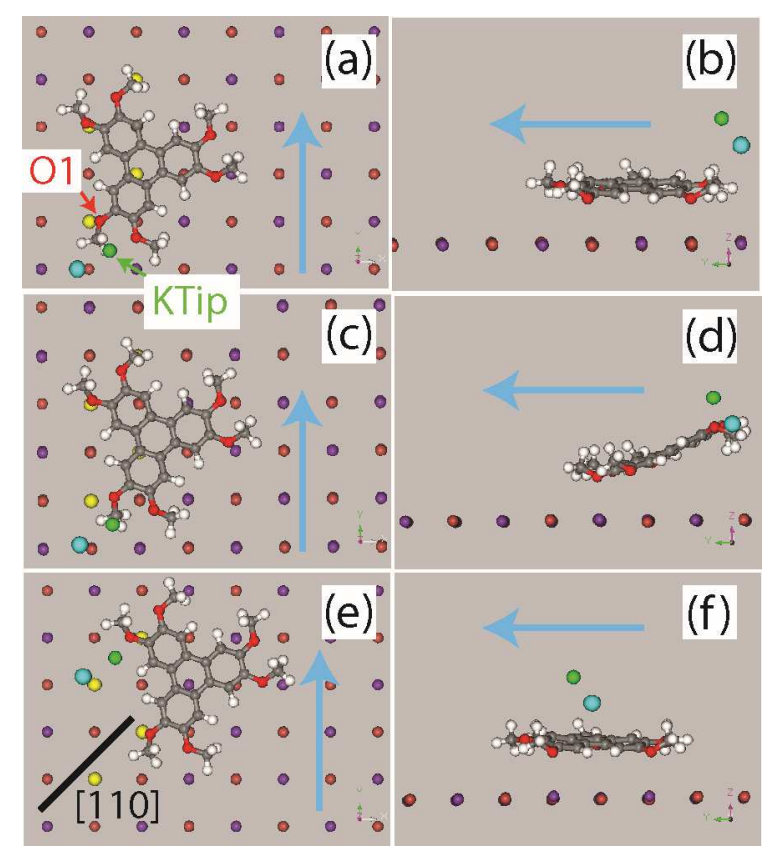

Figure 10: Images extracted from V1 and V2 (SI). (b), (d) and $(f)$ : Views along $+X$. For the sake of simplicity, the tip is represented by two of its ions only. The Br- ion terminating the tip is colored in blue, its $\mathrm{K}^{+}$ion is colored in green. The yellow $\mathrm{K}^{+}$ions are the initial and final binding sites of the molecule, The fast scanning direction is indicated by the blue arrows. $A=70 \mathrm{pm}, D=0.73 \mathrm{~nm}$.

Similar calculations with a $\mathrm{K}^{+}$tip show that in this case the molecule is manipulated at much larger tip-surface distance. The calculated sequence (V3 and V4 in SI) shows that even at 
large distance $($ closest approach $=\mathrm{D}-\mathrm{A}=0.98 \mathrm{~nm}$ ) the molecule can be picked up by the tip. The images of Figure 11 are extracted from this sequence.

The molecule is at first partially lifted up by the interaction of the $\mathrm{K}^{+}$terminating tip ion with one then two of the oxygen atoms (O5 and O4) of the molecule (Fig. 11b and c). It is then rotated by $90^{\circ}$ and translated along the scanning direction (Fig. 11d and e). After the inversion of the scanning direction, the molecule is pulled in the opposite direction and the two oxygen atoms interact now with a $\mathrm{K}^{+}$ion on the right side of the tip (Fig. $11 \mathrm{f}$ and g). Finally the molecule is picked up by the tip (Fig. 11h and i). Here also, as indicated in Figure 11c and i, the distances between $\mathrm{O} 4$ and $\mathrm{O} 5$ and the $\mathrm{K}+$ ions of the tip approaches the $\mathrm{O}-\mathrm{K}+$ equilibrium distance of $0.27 \mathrm{~nm}$, revealing the contribution of the repulsive short-range part of the van der Waals interaction.

This finding could explain why most of the experimental images we obtained are of the " $\mathrm{Br}^{-}$ tip" type: with $\mathrm{K}^{+}$tip the molecule is picked up before a clear contrast could be obtained.

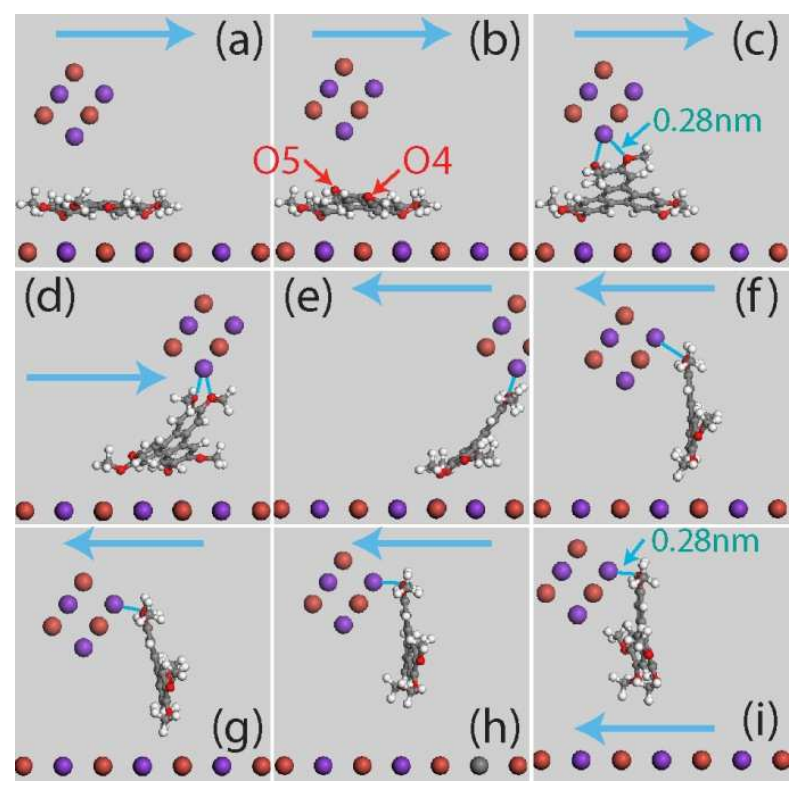

Figure 11: Images extracted from the $K^{+}$tip manipulation sequence V4 (SI). Views along $+X$. $A=110 \mathrm{pm}, D=1.09 \mathrm{~nm}$. The arrows indicate the scanning direction. Blue lines show where 
the electrostatic bonding between two oxygen atoms of the molecule and a $K+$ ion of the tip is located.

\section{Discussion and conclusions}

The comparison of the images of Figure 5, calculated with a $\mathrm{Br}^{-}$tip and the experimental images of Figure 2 shows a fair qualitative agreement. They display a near central clear protrusion, reaching frequency shifts that are higher than on $\mathrm{KBr}$, surrounded by two or more dark minima. Furthermore, the order of magnitude of $\Delta \mathrm{f}$ is comparable for the $\mathrm{D}$ range chosen for the calculations. In addition, the comparison with the images of Figure 6, obtained with a $\mathrm{K}^{+}$tip, clearly excludes a positive polarity tip. These findings lead to the following conclusions:

-The tip used in the experiment has a negative polarity

-The experimental tip surface distance is around $\mathrm{D}=1.1 \mathrm{~nm}$. For much smaller values of $\mathrm{D}$ (below $\sim 0.85 \mathrm{~nm}$ ), the molecule is manipulated while for much higher values (above $\sim 1.3 \mathrm{~nm}$ ), the $\Delta \mathrm{f}$ range becomes too small. In this range, the contrast is dominated by the electrostatic interaction as demonstrated in Figure 7.

These conclusions are very robust: they do not depend strongly on the size (Figure S2) and orientation of the tip (Figure S3). This robustness is obviously the consequence of the long range interactions that are involved in the image formation; the image is the result of a spatial average on a length scale given by the range of the interaction, i.e. nanometers in our case and is rather insensitive to atomic details.

These findings are also consistent with the simulations of the manipulation process with the two kinds of tips shown in the preceding part. These simulations highlight the important role played by the bounds that form between the oxygen atoms of the molecule and some $\mathrm{K}+$ ions of the tip, whatever the polarity of the tip. This is not surprising as the partial charge on the 
oxygen atom is by far the highest in the molecule (see Figure S1). The formation of these bounds means that in the manipulation process, the short-range repulsive contribution of the tip molecule potential plays an important role. The consequence is that, in contrast to the case of imaging, the atomic structure of the tip is expected to have a strong influence on the manipulation process.

The only way to improve the imaging resolution would be to reduce the tip-surface distance. But, in this situation, the molecule is not stable enough on the surface to resist the force applied by the tip. This limitation depends in a complex way on the structure of the moleculesurface system. Even if the adsorption energy is not so small $\left(\mathrm{E}_{0}=0.87 \mathrm{eV}\right)$, the flexibility of the triphenylene core provides low force paths to displace or pick up the molecule, as shown in Figure 10 and 11.

Nevertheless, the results of Figure 8 shows that the position of the methyl groups of the molecule have a noticeable influence. This result demonstrates that a precise adsorption structure determination should be possible from experimental images, even with a resolution limited by the long range nature of electrostatic forces.

\section{Supporting Information Description}

Figure S1: Atomic charges of HMTP

Figure S2: Influence of the tip size

Figure S3: Influence of the tip orientation

Figure S4: Time evolution of tip-molecule and molecule-surface distances during the manipulation sequence displayed in V1 and V2.

Video files V1.avi to V4.avi: Calculated manipulation sequences with a $\mathrm{Br}^{-}$tip and a $\mathrm{K}^{+}$tip. 
Acknowledgements: This work has been supported by the European Commission within the PAMS project (Contract $\mathrm{N}^{\circ}$ 610446).

\section{References}

(1) Burke, S. A.; Topple, J. M.; Grütter P. J. Phys. Condens. Matter 2009, 21, 423101.

(2) Lammle, K.; Trevethan, T.; Schwarz, A.; Watkins, M;. Shluger, A.; Wiesendanger, R. Nanoletters 2010, 10, 2965-2971.

(3) Such, B.; Trevethan, T.; Glatzel, T.; Kawai, S.; Zimmerli, L.; Meyer, E.; Shluger, A. L.; Amijs, C. H. M.; de Mendoza, P.; Echavarren, A. M. ACS nano 2010, 4, 3429-39.

(4) Trevethan, T.; Such, B.; Glatzel, T.; Kawai, S.; Shluger, A. L.; Meyer, E.; de Mendoza, P.; Echavarren, A. M. Small 2011, 7, 1264-1270.

(5) Hinaut, A.; Lekhal, K.; Aivazian G.; Bataillé, S.; Gourdon, A.; Martrou D.; Gauthier, S. J. Phys. Chem. C, 2011, 115, 13338-13342.

(6) Bocquet, F.; Nony, L.; Mannsfeld, S. C. B.; Oison, V.; Pawlak, R.; Porte, L.; Loppacher, Ch. Physical Review Letters 2012, 108, 6103.

(7) Hinaut, A., Pujol, A., Chaumeton, F., Martrou, D., Gourdon, A., Gauthier, S. Beilstein J. Nanotechnol. 2012, 3, 221.

(8) Kittelmann, M.; Nimmrich, M.; Neff, J. L.; Rahe P.; Gren, W; Bouju, X.; Gourdon, A.; Kühnle, A. J. Phys. Chem. C 2013, 117, 23868-23874.

(9) Gao, D. Z.; Grenz, J.; Watkins, M.B.; Canova, F.F.; Schwarz, A.; Wiesendanger, R.; Shluger A.L. ACS Nano, 2014, 8, 5339.

(10) Hoff, B.; Gingras, M.; Peresutti, R.; Henry, C. R.; Foster, A. S.; Barth, C. J. Phys. Chem. C, 2014, 118, 14569.

(11) Neff, J. L.; Milde, P.; Perez Leon, C.; Kundrat, M. D.; Eng, L. M.; Jacob, C. R.; Hoffmann-Vogel, R. ACS Nano 2014, 8, 3294. 
(12) Amrous A.; Bocquet F.; Nony L.; Para F.; Loppacher C.; Lamare S.; Palmino F.; Cherioux F.; Gao D. Z.; Canova F. F.; Watkins M. B.; Shluger A. L. Adv. Mater. Interfaces, 2014, $1,1400414$.

(13) Neff, J. L.; Songen, H.; Bechstein, R.; Maass, P.; Kuhnle, A. J.Phys.Chem.C 2015, 119, $24927-24931$.

(14) Documention of Materials Studio v5.5.0.0, Accelrys Software Inc.: San Diego, CA. http://accelrys.com/products/datasheets/compass.pdf

(15) Materials Studio v5.5.0.0, Accelrys Software Inc.: San Diego, CA.

(16) Sun, H. J. Phys. Chem. B 1998, 102, 7338-7364.

(17) Zhao, L.; Liu, L.; Sun, H. J. Phys. Chem. C 2007, 111, 10610-10617.

(18) Trevethan, T.; Shluger, A. L. J. Phys. Chem. C 2007, 111, 15375-15381.

(19) Gross, L.; Mohn, F.; Moll, N.; Liljeroth, P.; Meyer, G. Science 2009, 325, 1110-4.

(20) Pakarinen, O. H.; Barth, C.; Foster, A. S.; Henry C. R. Journal of Applied Physics, 2008, $103,054313$.

(21) Oyabu, N.; Pou, P.; Sugimoto, Y.; Jelinek, P.; Abe, M.; Morita, S.; Pérez, R.; Custance, O. Phys. Rev. Lett. 2006, 96, 106101.

(22) Barth, C.; Foster, A. S.; Reichling, M.; Shluger, A. L. J. Phys.: Condens. Matter 2001, 13, 2061-2079.

(23) Shluger, A. L.; Livshits, A. I.; Foster, A. S.; Catlow, C. R. A. J. Phys.: Condens. Matter 1999, 11, R295-R322.

(24) Giessibl, F. J., Phys. Rev. B 1997, 56, 16010.

(25) Teobaldi, G.; Lammle, K.; Trevethan, T.; Watkins, M.; Schwarz, A.; Wiesendanger, R.; Shluger, A. L. Phys. Rev. Lett. 2011, 106, 216102.

(26) Gross, L.; Schuler, B.; Mohn, F.; Moll, F.; Pavliček, N; Steurer, W.; Scivetti, I.; Kotsis, K.; Persson, M.; Meyer, G. Phys. Rev. B 2014, 90, 155455. 
(27) Bartels, L.; Meyer, G.; Rieder K.-H. Phys. Rev. Lett. 1997, 79, 697.

(28) Bouju, X.; Joachim, C.; Girard, C. Phys. Rev. B 1999, 59, R7845.

(29) Trevethan, T.; Kantorovich, L.; Polesel-Maris, J.; Gauthier, S. and Shluger, A. Phys. Rev. B 2007, 76, 085414. 
Table of Contents Graphics

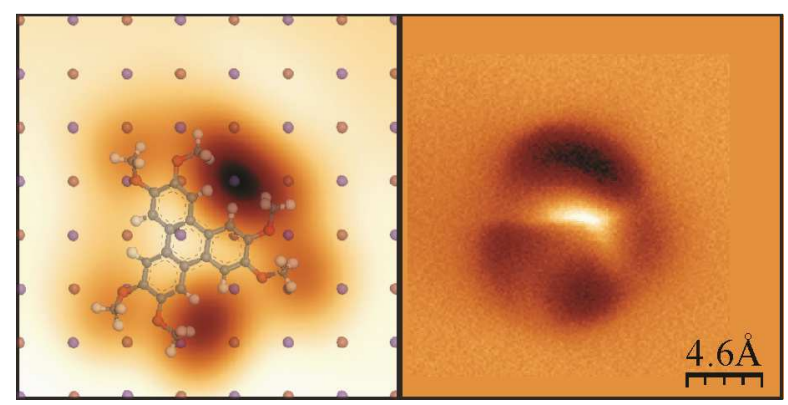




\section{Supporting Information}

\section{Atomic partial charges of HMTP}

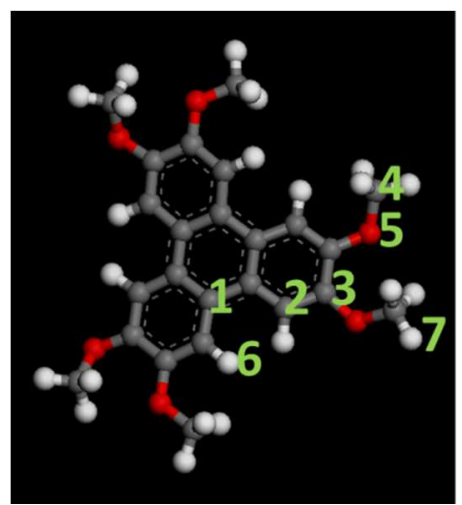

Partial charges:

(in |e| units)

$\mathrm{C} 1=0$

$\mathrm{C} 2=-0.127$

$\mathrm{C} 3=+0.042$

$\mathrm{C} 4=+0.001$

$\mathrm{O} 5=-0.202$

$\mathrm{H} 6=+0.127$

$\mathrm{H} 7=+0.053$

Figure S1: Atomic charges of HMTP as given by the COMPASS force-field.

\section{Influence of the tip size}

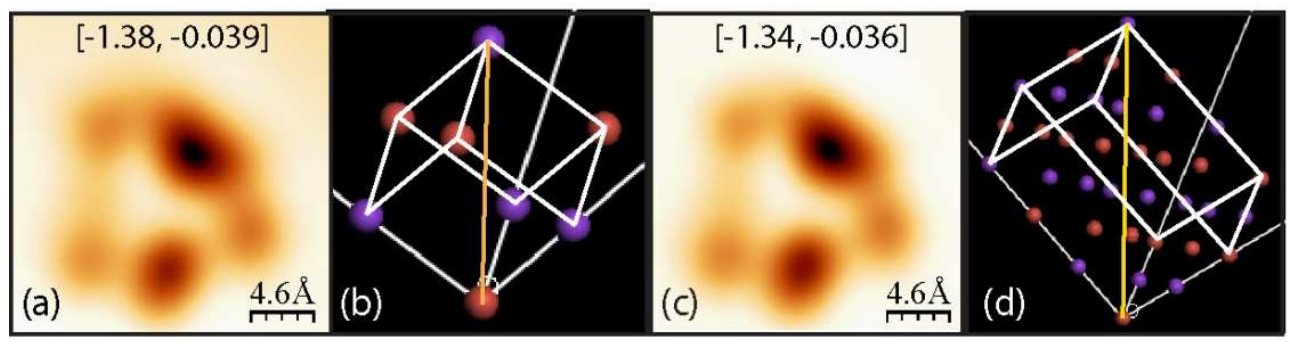

Figure S2: (a) $\Delta f$ image calculated with the Br- tip shown in (b). (c) $\Delta f$ image calculated with the Br tip shown in (d). D=11 .

\section{Influence of the tip orientation}

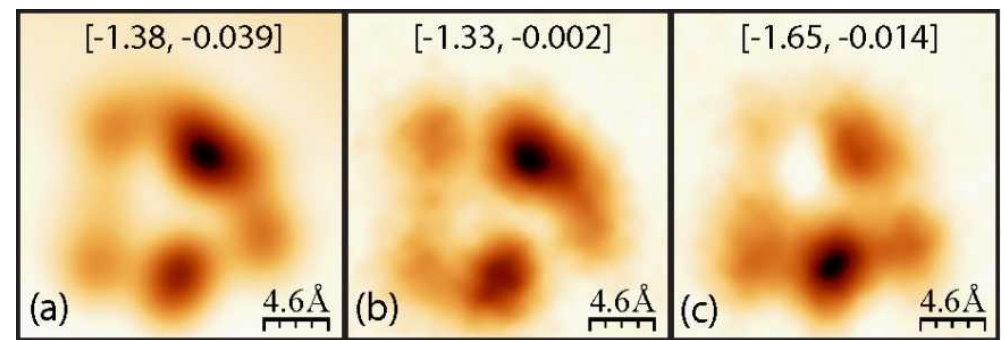

Figure S3: (a) $\Delta f$ image calculated with the non-rotated Br- tip, (b) with the Br- tip rotated by $45^{\circ}$, (c) with the Br tip tilted by $30^{\circ}$ in the vertical upward direction. $D=11 \AA$. 


\section{Manipulation with a Br- tip}

\section{Video files: V1.avi and V2.avi}

Legend: Top view and side view along $\mathrm{X}$ of a calculated manipulation sequence with the $\mathrm{Br}^{-}$ tip. $\mathrm{D}=0.73 \mathrm{~nm}, \mathrm{~A}=70 \mathrm{pm}$. The tip $\mathrm{Br}^{-}$ions is colored in blue. The surface $\mathrm{K}^{+}$ions on which the molecule is electrostatically bound at the beginning (end) of the sequence are colored in yellow (green). The $\mathrm{Z}$ oscillation of the tip is not displayed but only the frames where the tip is closest to the surface.

An inspection of V1 and V2 shows that the molecule interacts in a globally repulsive way with the two methoxy groups that include $\mathrm{O} 1$ and $\mathrm{O} 2$. The molecule "oscillate" (anticlockwise rotation on $\mathrm{O} 1$, clockwise rotation on $\mathrm{O} 2$ ) but stays in the same adsorption site until frame $\sim 5650$. The distances remain above $0.4 \mathrm{~nm}$, i.e. in the electrostatic range (Fig. S4b).

From frame $\sim 5650$ to frame $\sim 6300$, the system is in a transitory state during which the molecule is laterally displaced (Fig. S4d) . Fig. S4c shows that before the manipulation, the molecule is bound to the surface by $\mathrm{O} 1$ on $\mathrm{K} 1$ and $\mathrm{O} 2$ on $\mathrm{K} 2$, as shown by the red and green curves $(\mathrm{dO} 1 \mathrm{~K} 1$ and $\mathrm{dO} 2 \mathrm{~K} 2<0.35 \mathrm{~nm})$. After the manipulation, the molecule binds to the surface by $\mathrm{O} 2$ on $\mathrm{K} 3$ and $\mathrm{O} 1$ on $\mathrm{K} 5$ (pink and dark yellow curves, $\mathrm{dO} 2 \mathrm{~K} 3$ and $\mathrm{dO} 1 \mathrm{~K} 5<0.35$ $\mathrm{nm})$. ). It has moved by one nearest neighbor distance along $\langle 100\rangle$.

A jump occurs at frame 5684 (arrow 2 in Fig. S4 d and e). Fig. S4d shows that O1 gets closer to the tip (green and pink curves). Simultaneously O1 is lifted from the surface (Fig. S4e green curve). $\mathrm{O} 1$ is captured by the tip, lifting the extremity of the molecule from the surface (see also Fig. 10).

From frame $\sim 5700$ to frame $\sim 5900, \mathrm{O} 3$ and $\mathrm{O} 4$ get closer to $\mathrm{K} 3$ and $\mathrm{K} 4$ while $\mathrm{O} 1$ gets

farther from K1. From frame $\sim 5900$ to $\sim 6250$, the molecule binds to the surface by $\mathrm{O} 3$ on K3 (blue curve in Fig. S4e ) and O4 on K4 (pink curve in Fig.S4e ).

A final jump occurs near frame 6300 (arrow 3 in Fig. S4 d and e). $\mathrm{O} 2$ binds to K5 and O1 binds to K3. 

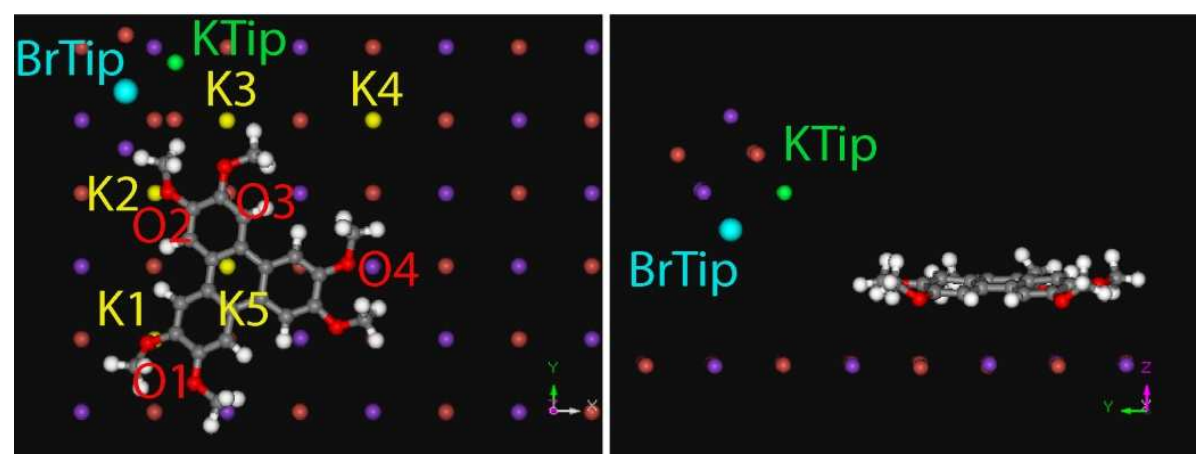

(a)

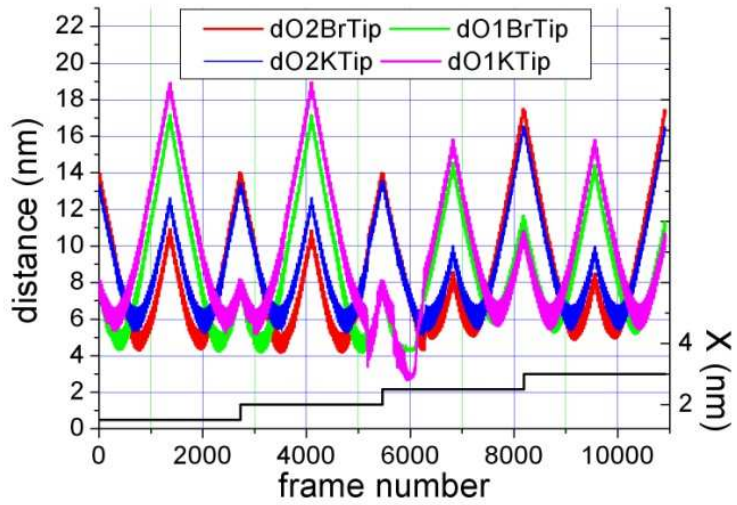

(b)

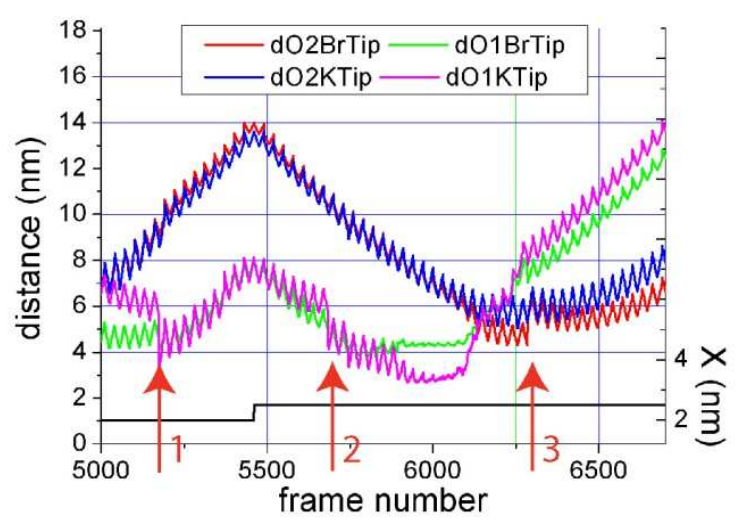

(d)

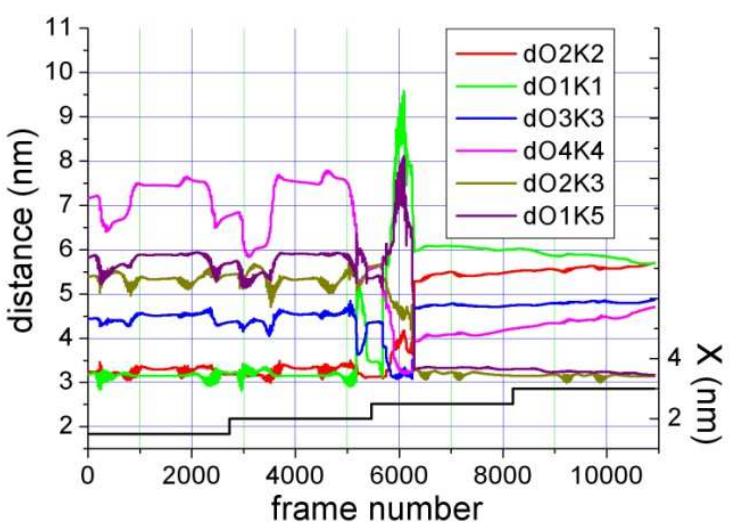

(c)

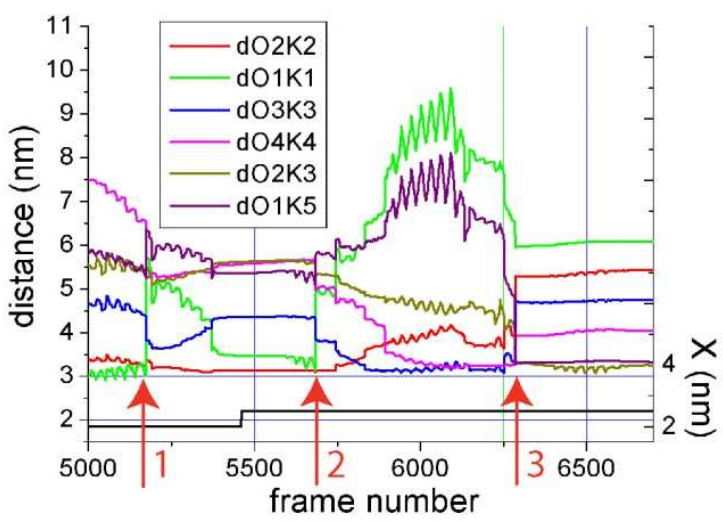

(e)

Figure S4: Time evolution during the manipulation sequence of videos V1 and V2 of the distances between selected $O$ atoms of the molecule and :

- (b) and (d) the terminating $B r$ - and one of the $K+$ of the tip

- (c) and (e) K1 to K5 K+ ions of the surface

$(d)$ and $(e)$ are enlargements of $(b)$ and $(c)$. See (a) for the labelling of the different species. 
Around frame $\sim 6000$, dO1-Ktip goes below $0.27 \mathrm{~nm}$. This is less that the sum of the ionic radii of $\mathrm{O}^{-}$and $\mathrm{K}^{+}$. It indicates that the $\mathrm{O} 1$ is strongly bound to the tip that pushes on the molecule as suggested by the images of Figure 10.

Note that these two jumps are preceded by an unsuccessful event (arrow 1 in Fig. S4 d and e): The molecule starts to move as immediately after jump 2 but goes back to its initial position (O1 on $\mathrm{K} 1$ and $\mathrm{O} 2$ on $\mathrm{K} 2$, red and green curves). It is only when $\mathrm{X}$ is larger by $0.1 \mathrm{~nm}$, i.e. when the tip is closer to the molecule that the manipulation will be successful.

\section{Manipulation with a $\mathbf{K}^{+}$tip}

V3.avi: Top view of a calculated manipulation sequence with the $\mathrm{K}^{+}$tip.

V4.avi: Side view (seen along $-X$ ) of the same manipulation sequence as in V3.avi. $A=110$ $\mathrm{pm}, \mathrm{D}=1.09 \mathrm{~nm}$. 